\title{
Downregulation of XBP1 decreases serous ovarian cancer cell viability and enhances sensitivity to oxidative stress by increasing intracellular ROS levels
}

\author{
GUI HONG ZHANG* , JIN YAN KAI*, MIAO MIAO CHEN, QIAN MA, AI LING ZHONG, SU HONG XIE, \\ HUI ZHENG, YAN CHUN WANG, YING TONG, YUAN TIAN, REN QUAN LU and LIN GUO \\ Department of Clinical Laboratory, Fudan University, Shanghai Cancer Center, Shanghai 200032, P.R. China
}

Received December 27, 2018; Accepted July 3, 2019

DOI: $10.3892 / \mathrm{ol} .2019 .10772$

\begin{abstract}
Interaction between endoplasmic reticulum (ER) stress and oxidative stress contributes to the occurrence and development of various types of cancer. The X-box-binding protein 1 (XBP1), which is an important transcription factor in ER stress-related pathways, has also been reported to serve a protective role against oxidative stress. However, the role of $\mathrm{XBP} 1$ in serous ovarian cancer (SOC) remains elusive. The aim of the present study was to explore the biological function of XBP1 in SOC cells under normal or oxidative stress conditions. The expression of XBP1 was downregulated in the SOC cell lines A2780 and HO8910 by lentivirus-mediated short hairpin RNA (shRNA). Cell proliferative ability was evaluated by cell colony formation and viability assays. The sensitivity of ovarian cancer cells to oxidative stress was evaluated using cell survival rate and apoptotic rate, determined by the Cell Counting Kit-8 assay and flow cytometry, respectively. Reactive oxygen species (ROS) levels were measured by flow cytometry and cell immunofluorescence using a dichlorodihydrofluorescein diacetate probe. The mRNA and protein expression levels were detected by fluorescence quantitative polymerase chain reaction and western blot analysis, respectively. The results demonstrated that XBP1 was overexpressed in SOC compared with normal ovarian epithelial cells, and that downregulation of XBP1 significantly reduced cell proliferative ability. In addition, the downregulation of XBP1 significantly enhanced the sensitivity of SOC cells to $\mathrm{H}_{2} \mathrm{O}_{2}$ by
\end{abstract}

Correspondence to: Professor Lin Guo or Professor Ren Quan Lu, Department of Clinical Laboratory, Fudan University, Shanghai Cancer Center, 270 Dong'An Road, Xuhui, Shanghai 200032, P.R. China

E-mail: guolin500@hotmail.com

E-mail: lurenquan@126.com

${ }^{*}$ Contributed equally

Key words: X-box-binding protein 1, oxidative stress, reactive oxygen species, serous ovarian cancer, unfolded protein response increasing the intracellular ROS levels. The phosphorylation level of the mitogen-activated protein kinase (MAPK) p38 decreased in the cells of the XBP1-knockdown group. These results indicated that XBP1 may serve a protective role against oxidative stress in SOC cells, and the underlying molecular mechanism may be associated with the downregulation of phosphorylated p38. Therefore, targeting XBP1 may act synergistically with ROS inducers in the treatment of SOC.

\section{Introduction}

Ovarian cancer is the leading cause of gynecological cancer-related deaths in the United States (1). As the most common subtype of ovarian cancer, high-grade serous ovarian cancer (SOC) is a highly lethal tumor with a 5-year survival rate of $<30 \%$ due to its severe resistance to platinum-based chemotherapy (2). Over the past decades, the efficacy of early diagnosis and treatment of SOC has improved (3), but little progress has been made in terms of overall survival.

Oxidative stress and reactive oxygen species (ROS) are closely associated with the occurrence and development of cancers $(4,5)$. Although a slight increase in the intracellular ROS level is necessary for rapid proliferation of cancer cells (6-8), excessive generation of intracellular ROS may induce cell death by damaging cellular DNA, lipids and proteins (9). At present, a number of anticancer drugs, including chemotherapeutic agents (10) and molecular targeted drugs (11), which may effectively eliminate cancer cells by inducing generation of ROS, have been applied in a clinical setting. Based on previous research, it may be hypothesized that enhancing the sensitivity of cancer cells to oxidative stress may improve the therapeutic effect of oxidative stress inducers.

$\mathrm{X}$-box-binding protein 1 (XBP1) is an important transcription factor in the unfolded protein response (UPR), which is activated by endoplasmic reticulum (ER) stress. During ER stress, XBP1 is subjected to unconventional splicing and converted to an active form, termed spliced XBP1 (XBP1s) (12). XBP1 is widely expressed in adult tissues, and it serves a key role in cardiac myogenesis and plasma cell differentiation $(13,14)$. In addition, XBP1s is crucial for cancer cell survival under microenvironment stress conditions, such as hypoxia (15). Overexpression of XBP1 has been observed 
in cancer tissues; high expression of XBP1 may enhance cancer cell proliferation and migration and confer drug resistance (16-18). Several studies have demonstrated that blocking UPR signals may lead to tumor cell growth arrest and apoptosis in a variety of malignant tumors $(19,20)$, which suggests that XBP1 may be a potential target for cancer therapy. In addition, an association between XBP1 and oxidative stress has been reported $(21,22)$, which indicates that XBP1 silencing may act synergistically with ROS inducers in cancer treatment.

Cubillos-Ruiz et al (23) first reported that the immunosuppressive effect of XBP1 in patients with ovarian cancer was mediated by dendritic cell dysfunction. However, the number of studies focusing on the expression of XBP1 in SOC is limited, and little is known regarding its potential role in ovarian cancer cells. To the best of our knowledge, the present study is the first to demonstrate that XBP1 is overexpressed in SOC cells and it is also the first to investigate the biological function of XBP1 in ovarian cancer cells under conditions of oxidative stress. In addition, the present study aimed to determine whether the downregulation of XBP1 may increase the sensitivity of ovarian cancer cells to oxidative stress, and whether the combination of XBP1 silencing and oxidative stress inducers may exert synergistic anti-SOC effects. The association between the downregulation of XBP1 and the expression of p-p38 was also investigated.

\section{Materials and methods}

Cell culture. The human SOC cell lines A2780, HO8910 and SKOV3 were acquired from the Type Culture Collection of the Chinese Academy of Sciences, and the normal ovarian epithelial cell line HOSEpiC was purchased from ScienCell Research Laboratories, Inc. Cells were cultured in RPMI-1640 medium supplemented with $10 \%$ fetal bovine serum and $1 \%$ penicillin-streptomycin (all from Gibco; Thermo Fisher Scientific, Inc.). 293T cells (The Cell Bank of Type Culture Collection of the Chinese Academy of Sciences) were cultured in DMEM high glucose medium (Gibco; Thermo Fisher Scientific, Inc.). All cell lines were cultured at $37^{\circ} \mathrm{C}$ with $5 \% \mathrm{CO}_{2}$.

Database analysis. The mRNA expression levels of XBP1 in 426 SOC and 88 normal ovarian tissue samples were analyzed using the Gene Expression Profiling Interactive Analysis database (GEPIA; gepia.cancer-pku.cn; version no. GEPIA2). The Student's $\mathrm{t}$-test was used to compare the mean values of two groups.

Establishment of stable cell lines. Short hairpin RNA (shRNA) sequences, as shown in Table I, were designed using the Sigma-Aldrich RNAi Design Service. For stable transfection, XBP1 (shXBP1-2 and shXBP1-3) and negative control (shCtrl) shRNA were embedded into the pLKO.1-puro vector (Addgene) at the AgeI and EcoRI sites. Subsequently, the recombined pLKO.1-puro vector and the packaging plasmid psPAX2 and pMD2.G (Addgene) were co-transfected into $293 \mathrm{~T}$ cells at a ratio of 4:3:1 using the Lipofectamine ${ }^{\circledR} 2000$ transfection reagent (Invitrogen; Thermo Fisher Scientific, Inc.) at $37^{\circ} \mathrm{C}$. At $48 \mathrm{~h}$, the supernatant containing lentiviral particles was collected, and the ovarian cancer cell lines were infected with the lentiviruses using polybrene (Sigma-Aldrich; Merck $\mathrm{KGaA}$ ) at a concentration of $8 \mathrm{mg} / \mathrm{ml}$ for $24 \mathrm{~h}$ at $37^{\circ} \mathrm{C}$.
Table I. Sequences of primers and shRNAs.

\begin{tabular}{ll}
\hline Gene/target & \multicolumn{1}{c}{ Sequence $\left(5^{\prime} \rightarrow 3^{\prime}\right)$} \\
\hline XBP1 & F: ATGGATTCTGGCGGTATT \\
& R: AAAGGGAGGCTGGTAAGG \\
FTH1 & F: TGAAGCTGCAGAACCAACGAGG \\
& R: GCACACTCCATTGCATTCAGCC \\
NQO1 & F: CCTGCCATTCTGAAAGGCTGGT \\
& R: GTGGTGATGGAAAGCACTGCCT \\
HMOX1 & F: CCAGGCAGAGAATGCTGAGTTC \\
& R: AAGACTGGGCTCTCCTTGTTGC \\
GAPDH & F: GAAGGTGAAGGTCGGAGTC \\
shXBP1-2 & R: GAAGATGGTGATGGGATTTC \\
shXBP1-3 & GACCCAGTCATGTTCTTCAAA \\
\end{tabular}

Stably infected cells were screened using $2 \mu \mathrm{g} / \mathrm{ml}$ puromycin (Sigma-Aldrich; Merck KGaA) for 3-5 days, and the knockdown efficiency of the shRNA was determined by western blotting and reverse transcription-quantitative polymerase chain reaction (RT-qPCR) analysis.

$R T-q P C R$. Total RNA was extracted from cultured cells with TRIzol ${ }^{\circledR}$ reagent (Invitrogen; Thermo Fisher Scientific, Inc.) and reverse-transcribed into cDNA with the Prime Script ${ }^{\circledR}$ RT Reagent kit (Takara Bio, Inc.) according to the manufacturer's protocol. qPCR was performed in a $10-\mu 1$ reaction solution containing $20 \mathrm{ng}$ cDNA, $0.4 \mu \mathrm{l}$ forward primer, $0.4 \mu \mathrm{l}$ reverse primer and $5 \mu 12 \mathrm{X}$ SYBR Premix Ex Taq buffer (Takara Bio, Inc.). The PCR amplification was performed at $95^{\circ} \mathrm{C}$ for $5 \mathrm{~min}$, followed by 40 cycles of $95^{\circ} \mathrm{C}$ for $15 \mathrm{sec}$ and $65^{\circ} \mathrm{C}$ for $40 \mathrm{sec}$, using an ABI PRISM Detection System (Applied Biosystems; Thermo Fisher Scientific, Inc.). All experiments were performed in triplicate and normalized to GAPDH mRNA expression levels. mRNA levels were calculated using the $2^{-\Delta \Delta \mathrm{Cq}}$ method (24). The primer sequences are presented in Table I.

Western blot analysis. The cell lines HOSEpiC, A2780, HO8910 and SKOV3 were lysed on ice with cell lysis buffer IP (Beyotime Institute of Biotechnology) containing protease inhibitors for total protein extraction. Western blotting was performed as previously described (3). Protein expression levels were evaluated using enhanced chemiluminescence ECL reagent (Gene Tech Co., Ltd.). Densitometric analysis was performed using ImageJ software (version 1.8.0; National Institutes of Health) and normalized to the internal control $\beta$-actin. The primary antibodies used were as follows: XBP1 (1:1,000; rabbit polyclonal; cat. no. ab198999; Abcam), HMOX1 (1:500; mouse monoclonal; cat. no. sc-136960; Santa Cruz Biotechnology, Inc.), p-p38 (1:1,000; rabbit monoclonal; cat. no. D3F9; Cell Signaling Technology, Inc.), p38 (1:1,000; rabbit monoclonal; cat. no. 3042S; Cell Signaling Technology, Inc.), p53 (1:500; mouse monoclonal; cat. no. sc-126; Santa Cruz Biotechnology, Inc.), Bcl2 (1:1,000, rabbit polyclonal; cat. no. 12789-1-AP; ProteinTech Group, Inc.) and $\beta$-actin (1:5,000; 


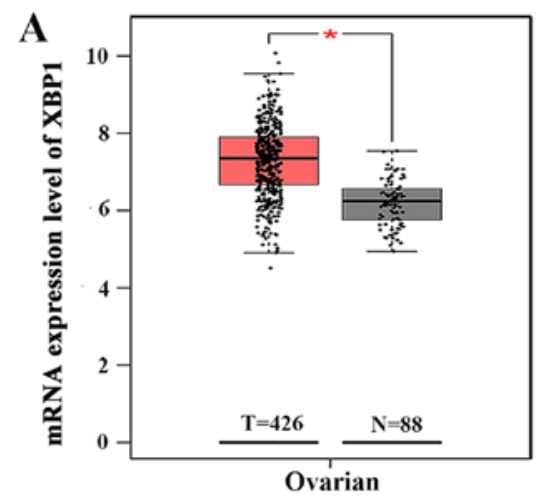

B
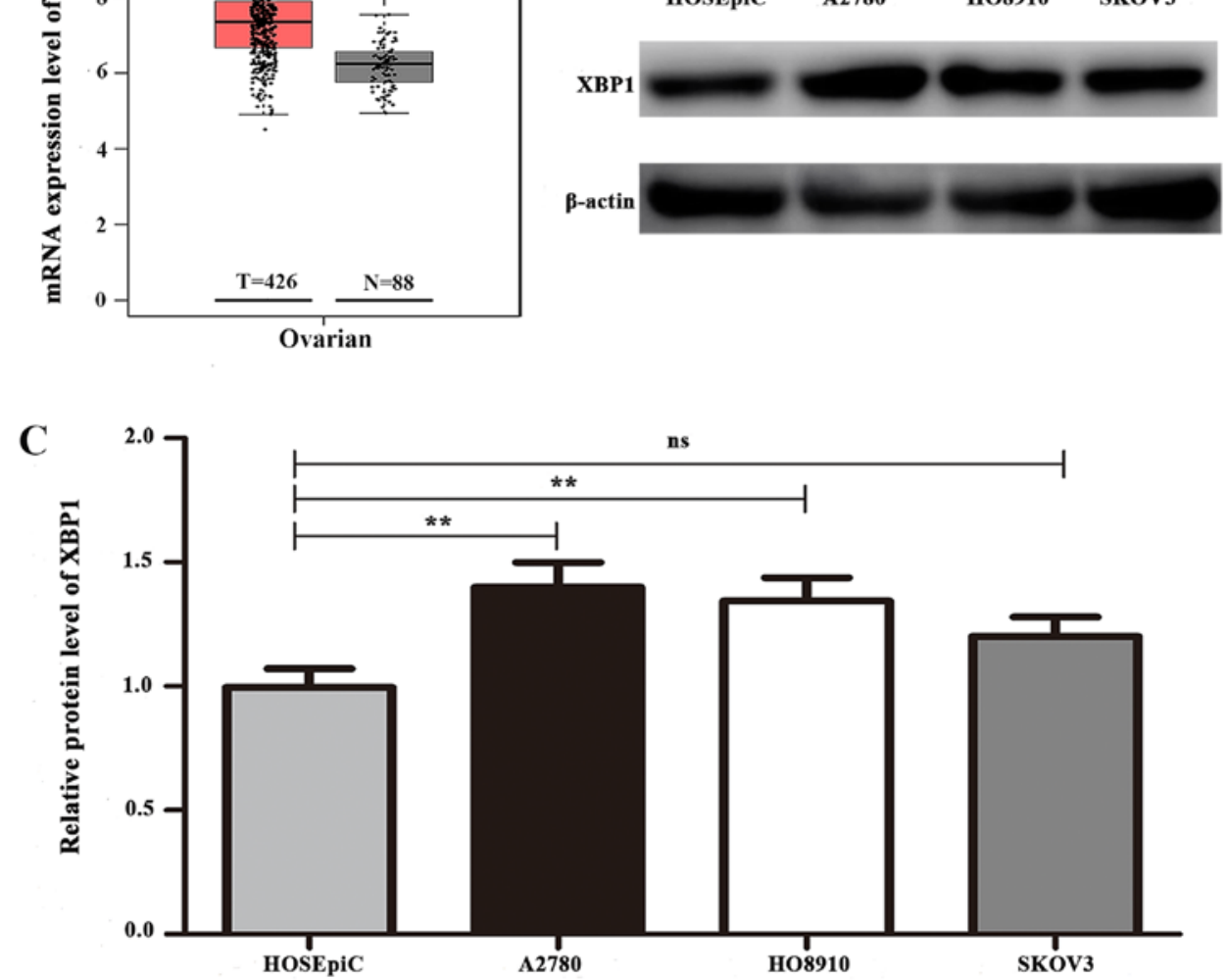

Figure 1. XBP1 expression is significantly increased in serous ovarian cancer. (A) Database analysis of XBP1 mRNA levels in ovarian cancer tissues and healthy controls. (B) Protein expression level of XBP1 in the normal ovarian epithelial cell line HOSEpiC and the ovarian cancer cell lines A2780, HO8910 and SKOV3. (C) Densitometric analysis of XBP1 protein expression in serous ovarian cancer cells. Student's t-test was used to determine the statistical differences between two groups. One-way analysis of variance and Tukey's test were performed to determine statistical differences between multiple groups. Data are presented as the mean \pm standard error of the mean. ${ }^{*} \mathrm{P}<0.05$ and ${ }^{* *} \mathrm{P}<0.01$. XBP1, X-box-binding protein 1; T, Tumor; N, Normal.

mouse monoclonal; cat. no. 60008-1-Ig; ProteinTech Group, Inc.). The secondary antibodies were as follows: Anti-mouse IgG-HRP (1:5,000; goat polyclonal; cat. no. sc-2005; Santa Cruz Biotechnology, Inc.), anti-rabbit IgG-HRP (1:5,000; goat polyclonal; cat. no. sc-2004; Santa Cruz Biotechnology, Inc.).

Hydrogen peroxide $\left(\mathrm{H}_{2} \mathrm{O}_{2}\right)$ treatment. A2780 cells were treated with 30 and $40 \mu \mathrm{M} \mathrm{H}_{2} \mathrm{O}_{2}$ for 24 and $48 \mathrm{~h}$ according to the different experimental purposes at $37^{\circ} \mathrm{C}$. The HO8910 cells were treated with 100 and $200 \mu \mathrm{M} \mathrm{H}_{2} \mathrm{O}_{2}$ for 24 and $48 \mathrm{~h}$ at $37^{\circ} \mathrm{C}$.

Colony formation and cell proliferation assays. For the colony formation assay, 1,000 cells/well were seeded into 6-well plates and incubated for 10-14 days, until colonies were visible. Cells were washed twice with PBS, fixed with $4 \%(\mathrm{w} / \mathrm{v})$ paraformaldehyde (Sigma-Aldrich; Merck KGaA) for $10 \mathrm{~min}$ at $4^{\circ} \mathrm{C}$ and stained with $0.5 \%$ crystal violet solution for $30 \mathrm{~min}$ at room temperature. The number of colonies containing $>50$ cells was counted using an optical microscope. To measure cell proliferation, $2 \times 10^{3}$ cells/well were seeded into 96 -well plates and incubated for $1,2,3,4$ and 5 days at $37^{\circ} \mathrm{C} ; 10 \mu \mathrm{l} \mathrm{Cell}$ Counting Kit-8 solution (Dojindo Molecular Technologies, Inc.) was added into each well and incubated for $2 \mathrm{~h}$ at $37^{\circ} \mathrm{C}$. The absorbance of each well at $450 \mathrm{~nm}$ was determined using a Synergy H4 microplate reader (Bio-Tek Instruments, Inc.).
Apoptosis assay. For apoptosis analysis, 3.0x $10^{5}$ cells/well were harvested from 6-well-plates, washed twice with PBS, re-suspended in $500 \mu \mathrm{l} 1 \mathrm{X}$ binding buffer and incubated with $5 \mu \mathrm{l}$ Annexin V-FITC/PI (BD Biosciences) for $15 \mathrm{~min}$ at $4^{\circ} \mathrm{C}$. Apoptotic rates were evaluated using flow cytometry.

Immunofluorescence. A total of $4.0 \times 10^{4}$ cells/well were fixed with $4 \%(\mathrm{w} / \mathrm{v})$ paraformaldehyde for $30 \mathrm{~min}$ at $4^{\circ} \mathrm{C}$ and auto-fluorescence was quenched with BSA. Subsequently, cells were stained with primary antibodies against XBP1 (1:200; rabbit polyclonal; cat. no. ab198999; Abcam) overnight at $4^{\circ} \mathrm{C}$, followed by incubation with secondary antibodies (Alexa Fluor-594; 1:1,000; cat. no. R37117; Thermo Fisher Scientific, Inc.) for $1 \mathrm{~h}$ at $37^{\circ} \mathrm{C}$. The cells were mounted with ProLong ${ }^{\circledR}$ Gold Antifade Reagent with DAPI (Thermo Fisher Scientific, Inc.) and visualized using a LAS-AF-Lite multi-photon confocal microscope system (Leica Microsystems, Inc.).

ROS detection. Intracellular ROS levels were determined by the fluorescence intensity of the dichlorodihydrofluorescein diacetate (DCFH-DA) probe. Cells were incubated with $10 \mathrm{mM}$ DCFH-DA for $30 \mathrm{~min}$ at $37^{\circ} \mathrm{C}$. Following two washes with ice-cold PBS, the cells were harvested for immediate detection by flow cytometry. The fluorescence intensity of cells was also observed under a multi-photon confocal microscope system. 
A
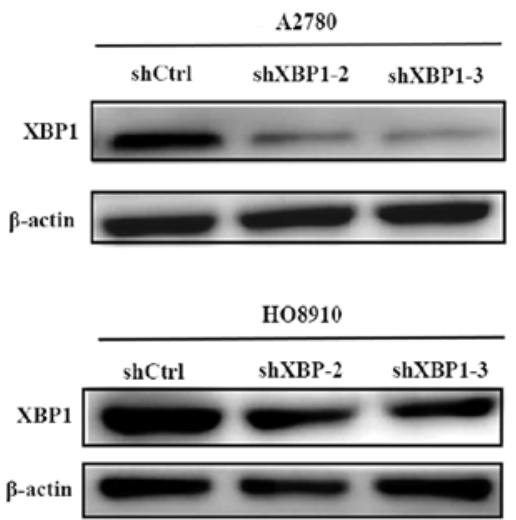

C
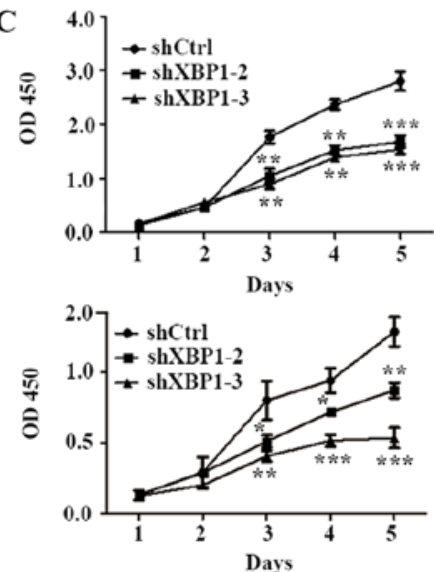
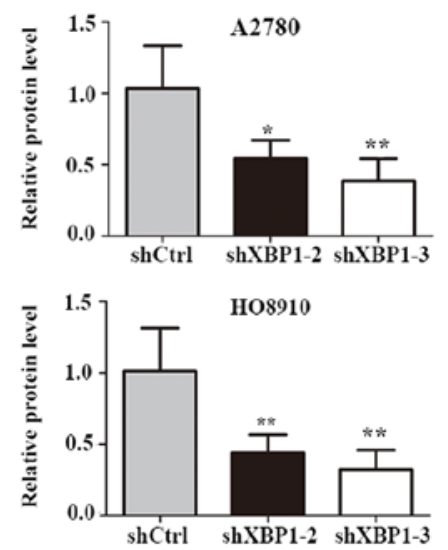

D
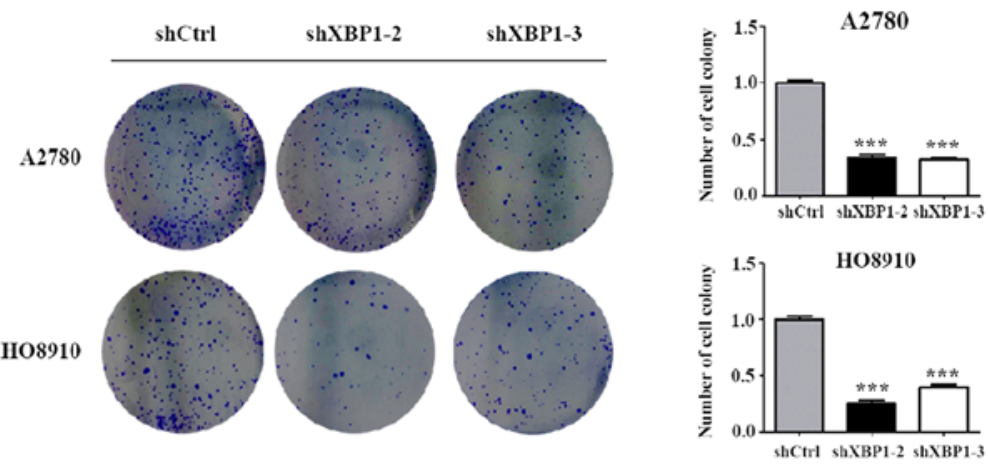

Figure 2. XBP1 downregulation suppresses the proliferation of serous ovarian cancer cells. (A and B) The efficiency of downregulation of XBP1 was determined by (A) western blot analysis and (B) RT-qPCR analysis in A2780 and HO8910 cells. (C and D) The downregulation of XBP1 significantly inhibited the proliferation of A2780 and HO8910 cells, as determined by (C) Cell Counting Kit-8 and (D) colony formation assay. One-way analysis of variance and Tukey's test were performed to determine statistical differences between multiple groups. Data are presented as the mean \pm standard error of the mean. "P<0.05, ${ }^{* *} \mathrm{P}<0.01$ and ${ }^{* * *} \mathrm{P}<0.001$ vs. shCtrl. XBP1, X-box-binding protein 1; RT-qPCR, reverse transcription-quantitative polymerase chain reaction.

Statistical analysis. The data are expressed as the mean \pm standard error of the mean of three independent experiments. Statistical analysis was performed using SPSS 19.0 software (IBM Corp.). Student's t-test was used to compare the mean values of two groups. One-way analysis of variance was used to compare the mean values of multiple groups with equal variances and Tukey's test was used to perform multiple comparisons as a post hoc test. $\mathrm{P}<0.05$ was considered to indicate statistically significant differences.

\section{Results}

$X B P 1$ expression is increased in SOC. To investigate the role of XBP1 in SOC, the mRNA expression levels of XBP1 in 426 SOC and 88 normal ovarian tissue samples were analyzed using the GEPIA database. The results revealed that the mRNA expression level of XBP1 in SOC tissues was significantly higher compared with that in normal ovarian tissues (Fig. 1A). For further verification, the protein expression level of XBP1 was detected in three SOC cell lines and the normal ovarian epithelial cell line HOSEpiC. Western blotting revealed a higher expression level of XBP1 in the A2780 and HO8910 ovarian cancer cells compared with that in HOSEpiC (Fig. 1B), whereas no increase in XBP1 expression was observed in SKOV3 cells. Densitometric analysis demonstrated significant differences in the protein expression levels of XBP1 between HOSEpiC and A2780 and HO8910 cells ( $\mathrm{P}<0.05$; Fig. 1C). Therefore, A2780 and HO8910 cells were selected for further experiments involving downregulation of XBP1 expression to investigate its biological function in ovarian cancer.

XBP1 downregulation inhibits cell proliferation. To investigate the potential functions of XBP1 in SOC cells, shXBP1-2/shXBP1-3/shCtrl lentiviral infection was performed in A2780 and HO8910 cells. To confirm that XBP1 was effectively downregulated by shRNA, RT-qPCR and western blotting were used to evaluate XBP1 mRNA and protein expression levels, respectively. Western blot analysis revealed that the protein level of XBP1 was significantly decreased by shXBP1-2 and shXBP1-3 in the two cell lines (Fig. 2A), and the results of qPCR demonstrated that shXBP1-2 and shXBP1-3 significantly decreased the mRNA level of XBP1 (Fig. 2B). Decreased levels of endogenous XBP1 were associated with inhibition of cell proliferation (Fig. 2C) and colony formation (Fig. 2D).

XBP1 downregulation enhances cell sensitivity to oxidative stress. To determine whether XBP1 protects SOC cells against oxidative stress, A2780 and HO8910 cells were treated with $\mathrm{H}_{2} \mathrm{O}_{2}$. Under the oxidative stress induced by $\mathrm{H}_{2} \mathrm{O}_{2}$, the mRNA 
A

A2780

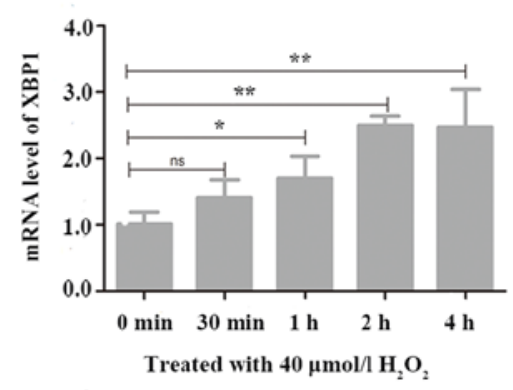

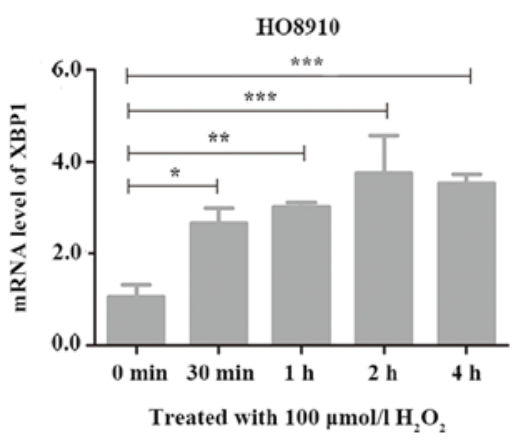

Treated with $100 \mu \mathrm{mol} / / \mathrm{H}_{2} \mathrm{O}_{2}$
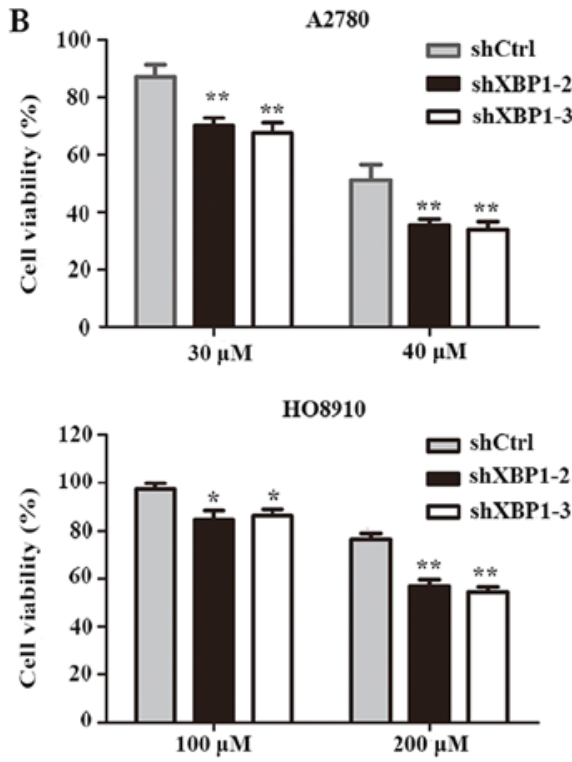

C $\mathbf{A 2 7 8 0}$

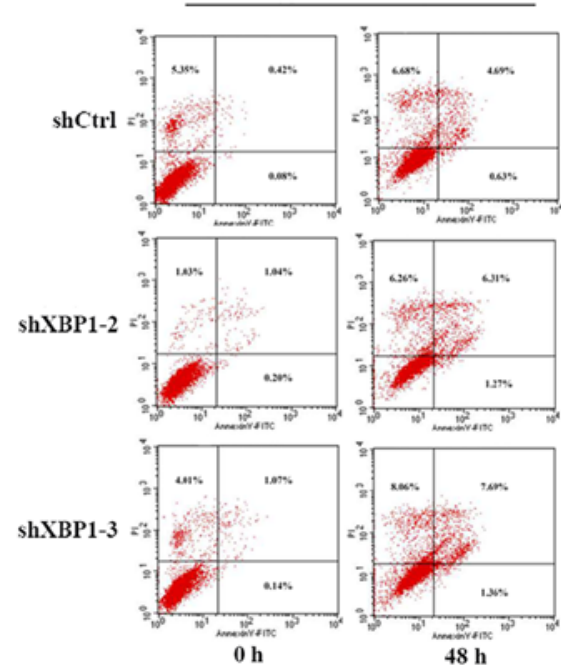

HO8910
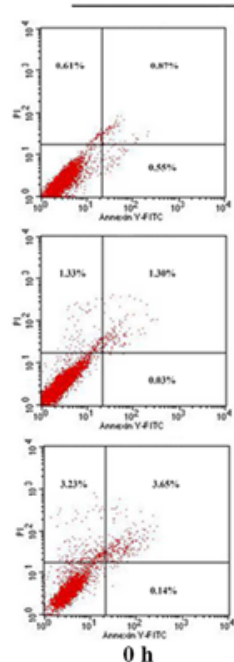
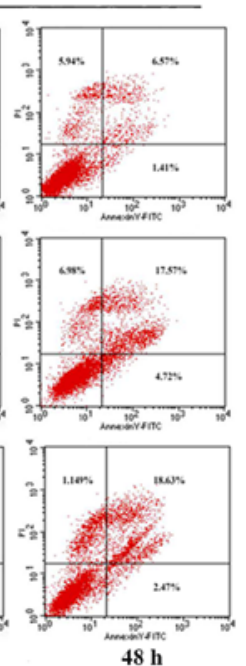

$\mathbf{A 2 7 8 0}$
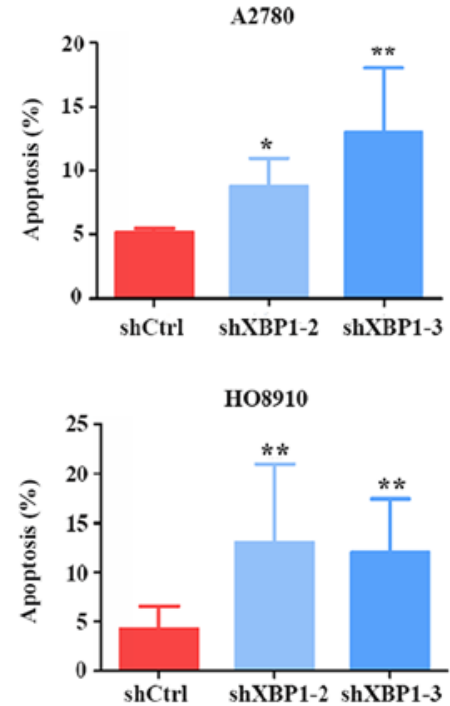

Figure 3. XBP1 downregulation enhances cell sensitivity to oxidative stress. (A) The expression of XBP1 was increased in serous ovarian cancer cells during treatment with $\mathrm{H}_{2} \mathrm{O}_{2}$. Upper panel, XBP1 mRNA expression level; lower panel, XBP1 protein expression level. One-way analysis of variance and Tukey test were performed to determine statistical differences between multiple groups. (B) Cell survival rates following $\mathrm{H}_{2} \mathrm{O}_{2}$ treatment in the knockdown groups were significantly decreased compared with that of the control group. Upper panel, A2780 cells; lower panel, HO8910 cells. (C) Apoptotic rates of cells in the knockdown groups were significantly increased compared with those of the control groups following treatment with $\mathrm{H}_{2} \mathrm{O}_{2}$. One-way analysis of variance and Tukey's test were performed to determine statistical differences between multiple groups. Data are presented as the mean \pm standard error of the mean. ${ }^{*} \mathrm{P}<0.05$, ${ }^{* * *} \mathrm{P}<0.01$ and ${ }^{* * *} \mathrm{P}<0.001$ vs. shCtrl or as indicated. XBP1, X-box-binding protein 1.

and protein expression levels of XBP1 gradually increased over time (Fig. 3A). A2780 cells were treated with 30 and $40 \mathrm{uM}$ $\mathrm{H}_{2} \mathrm{O}_{2}$ and $\mathrm{HO} 8910$ cells were treated with 100 and $200 \mathrm{uM}$ $\mathrm{H}_{2} \mathrm{O}_{2}$ for $24 \mathrm{~h}$. XBP1 downregulation significantly reduced the proliferative ability of cells compared with shCtrl (Fig. 3B). Following treatment with $\mathrm{H}_{2} \mathrm{O}_{2}$ for 48 h, A2780 cells were treated with $20 \mathrm{uM} \mathrm{H}_{2} \mathrm{O}_{2}$ and $\mathrm{HO} 8910$ cells were treated with $100 \mathrm{uM} \mathrm{H}_{2} \mathrm{O}_{2}$, the apoptotic rates were significantly increased in the XBP1-knockdown groups compared with those in the respective control groups (Fig. 3C). These results suggested that downregulation of XBP1 may enhance the sensitivity of ovarian cancer cells to oxidative stress.

XBP1 downregulation increases intracellular ROS levels. To investigate whether XBP1 is involved in the regulation of the intracellular ROS level, the intracellular ROS levels were measured in the XBP1-knockdown and control groups. The downregulation of XBP1 resulted in a significant increase in intracellular ROS levels in A2780 and HO8910 cells compared with shCtrl. ROS levels, as detected by flow cytometry, were increased in the A2780 and HO8910 cells of the knockdown group compared with the shCtrl group (Fig. 4A). Cell immunofluorescence was also performed to detect the intracellular ROS level in A2780 and HO8910 cells, and the results were consistent with those of flow cytometry (Fig. 4B).

$X B P 1$ downregulation induces changes in heme oxygenase 1 (HMOX1) and phosphorylated ( $p$-)p38 expression. To identify the underlying molecular mechanisms of XBP1-dependent ROS sensitivity regulation in SOC cells, the mRNA levels of 
A
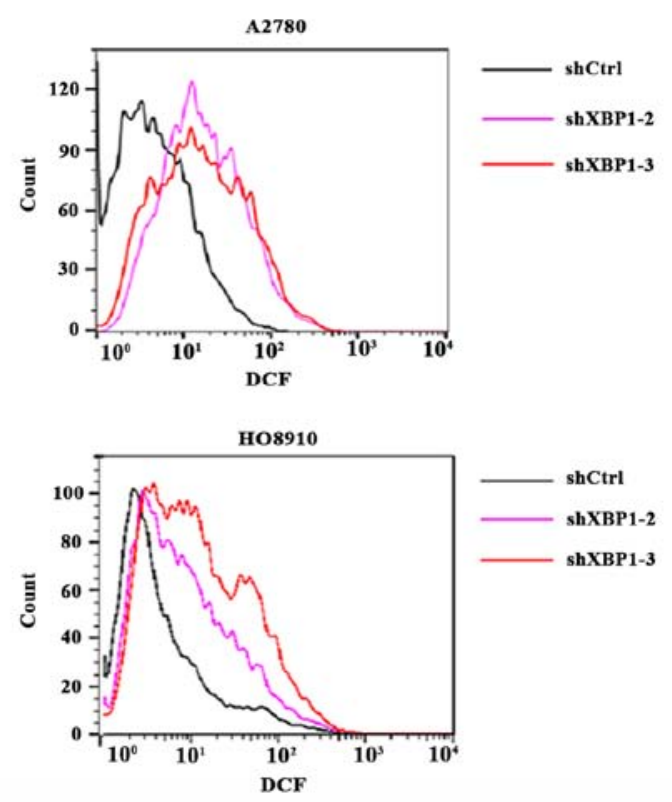
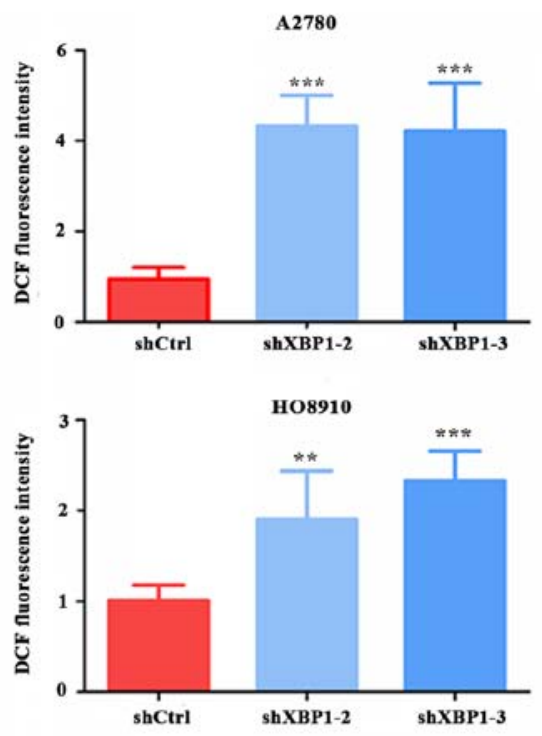

DAPI
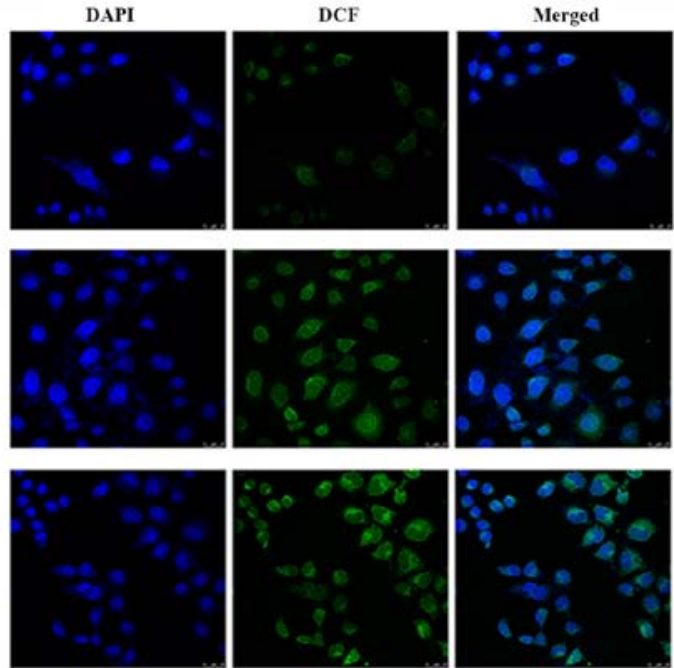

HO8910

Figure 4. XBP1 downregulation increases intracellular ROS levels. (A) Flow cytometry demonstrated that the intracellular ROS levels in cells in the knockdown groups were significantly higher compared with that in the control group in A2780 and HO8910 cells (B) The results of cell immunofluorescence (magnification, x50) were consistent with those of flow cytometry analysis. One-way analysis of variance and Tukey's test were performed to determine statistical differences between multiple groups. Data are presented as the mean \pm standard error of the mean. ${ }^{* *} \mathrm{P}<0.01$ and ${ }^{* * *} \mathrm{P}<0.001$ vs. shCtrl. $\mathrm{XBP} 1, \mathrm{X}$-box-binding protein 1 ; ROS, reactive oxygen species.

the antioxidant genes ferritin heavy chain 1 (FTH1), quinine oxidoreductase (NQO1) and HMOX1, which are regulated by nuclear factor erythroid 2 like 2 (Nrf2), were evaluated by RT-qPCR. The expression levels of these genes were increased to a variable extent in A2780 cells of the knockdown group; HMOX1 was also increased in HO8910 cells with downregulated XBP1 expression (Fig. 5A). The results of western blotting further confirmed that HMOX1 expression was increased in the knockdown group (Fig. 5B). The results indicated that the downregulation of XBP1 increased antioxidant production in ovarian cancer. The expression levels of the antioxidant genes FTH1, NQO1 and HMOX1 in the Nrf2 signaling pathway were increased to varying degrees, and HMOX1 was significantly upregulated following XBP1 knockdown. However, the increased intracellular ROS levels induced by the downregulation of XBP1 may stimulate the expression of these antioxidant genes in response. Detection of apoptosis-related proteins demonstrated that P53, Bcl2 and total p38 expression levels did not change in the knockdown group, whereas the phosphorylation level of the stress kinase p38 was decreased compared with shCtrl (Fig. 5C).

\section{Discussion}

To the best of our knowledge, the present study is the first to demonstrate that XBP1 is overexpressed in A2780 and HO8910 SOC cells, and that the downregulation of XBP1 in A2780 and HO8910 cells may result in decreased cell viability. Oxidative stress may stimulate the expression of XBP1 in SOC cells. In addition, the downregulation of XBP1 may significantly 
$\mathbf{A}$

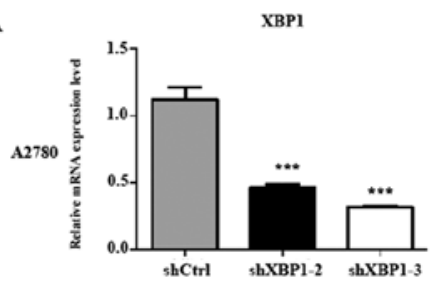

XBP1

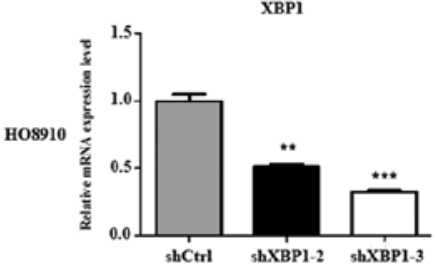

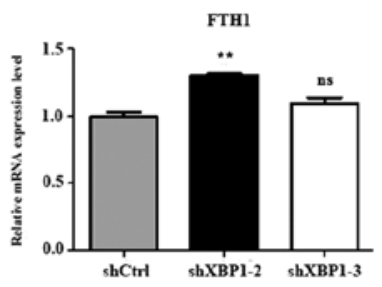

FTHI

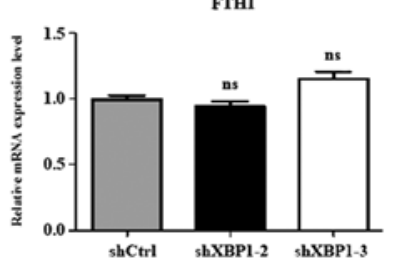

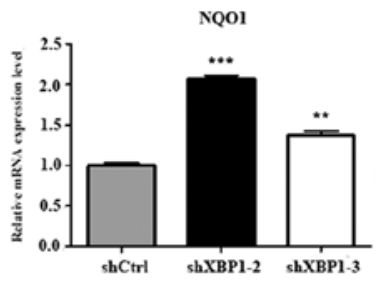

NQOI

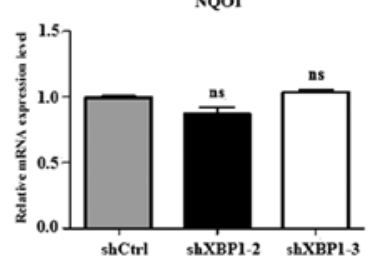

HMOXı

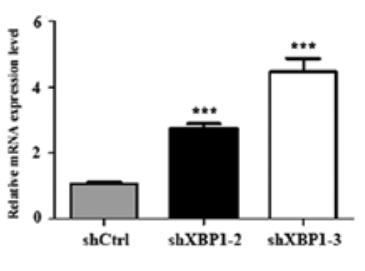

mMoxı

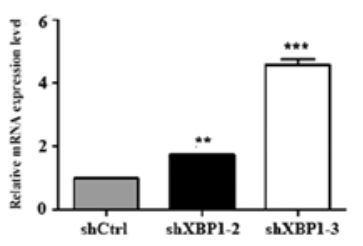

B

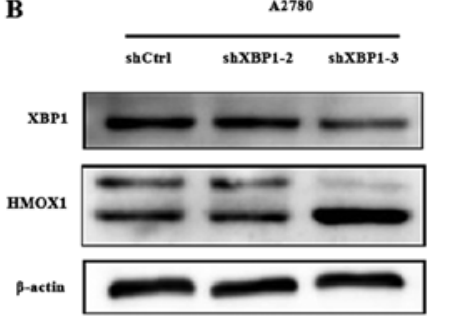

C

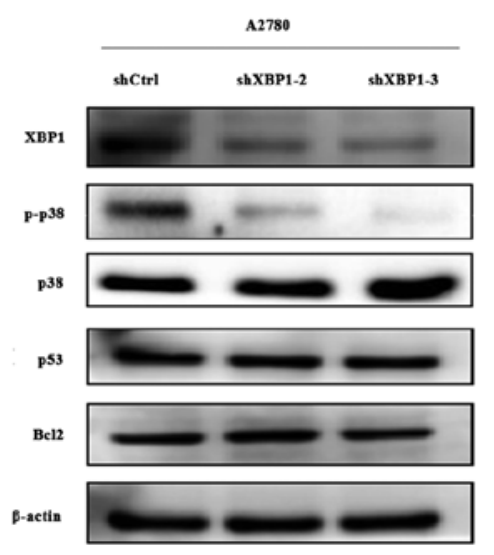

нов910

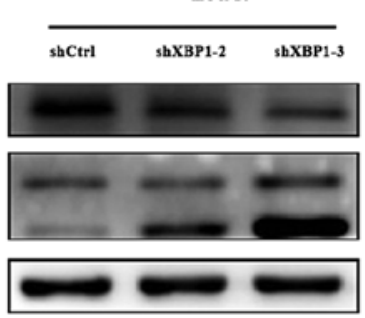

A2780

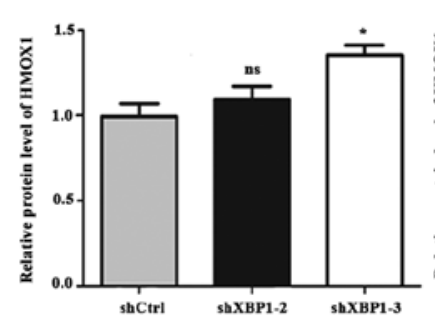

но8910

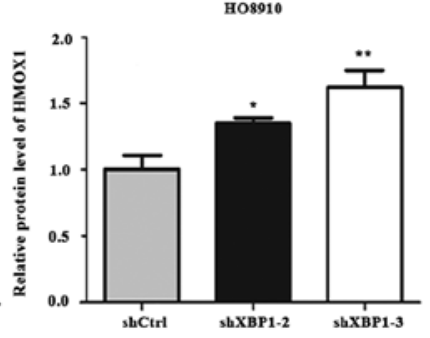

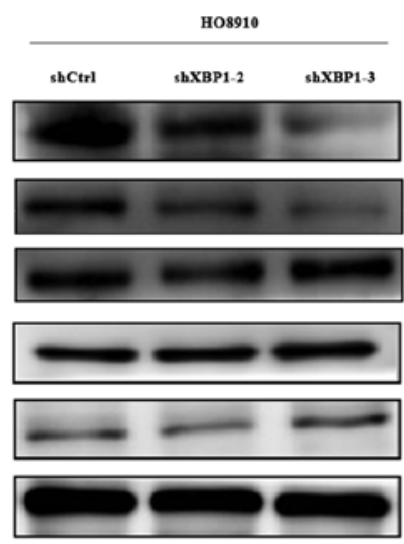
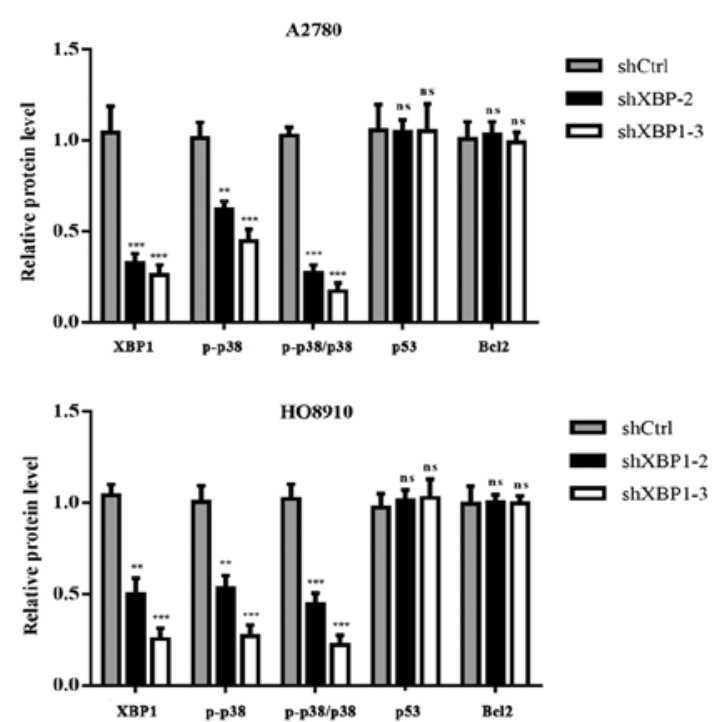

Figure 5. XBP1 downregulation induces changes in HMOX1 and p-p38 expression. (A) The results of qPCR demonstrated that the downregulation of XBP1 led to the upregulation of antioxidant genes FTH1, NQO1 and HMOX1 to varying degrees in A2780 cells. (B and C) The western blotting results revealed that compared with the control groups, (B) HMOX1 was increased in the knockdown group of A2780 and HO8910 cells and (C) the phosphorylation levels of the stress kinase p38 were decreased in the knockdown group, whereas no changes in the expression of p53 and Bcl2 were observed. One-way analysis of variance and Tukey's test were performed to determine statistical differences between multiple groups. Data are presented as the mean \pm standard error of the mean. ${ }^{*} \mathrm{P}<0.05,{ }^{* * *} \mathrm{P}<0.01$ and ${ }^{* * * *} \mathrm{P}<0.001$ vs. shCtrl. XBP1, X-box-binding protein 1 ; HMOX1, heme oxygenase 1 ; qPCR, quantitative polymerase chain reaction; NQO1, quinine oxidoreductase; FTH1, ferritin heavy chain 1.

enhance the sensitivity of SOC cells to oxidative stress through the induction of ROS generation. These results suggested that XBP1 may serve an important role in promoting ovarian cancer cell proliferation and exert protective effects in SOC cells against oxidative stress. Downregulation of XBP1 markedly increased the expression of HMOX1 in A2780 and HO8910 cells; the expression levels of FTH1 and NQO1 were also increased in A2780 cells. The results obtained in the current study indicated that the downregulation of XBP1 increased antioxidant production. The increased intracellular ROS level caused by the downregulation of XBP1 may stimulate the expression of antioxidant genes. To further investigate the mechanism of XBP1 is involvement in the resistance of ovarian cancer cells to oxidative stress, the expression levels of the stress-related kinase p-p38 and the apoptosis-related proteins p53 and $\mathrm{Bcl} 2$ were detected. The results demonstrated that the downregulation of XBP1 decreased the phosphorylation levels of the stress kinase p38, but did not affect the expression of P53, Bcl2 and total p38. 
ER stress and the UPR pathway serve an important role in the occurrence and development of various tumors (25). Oncogene activation, hypoxia and nutrient deprivation induce ER stress in cancer cells $(26,27)$, and the activation of UPR in a stress environment is crucial for cancer cell growth and survival $(28,29)$. The initial activation of UPR may restore the normal function of ER through various signaling pathways, and a variety of molecules, such as HMOX1 and the MAPK family member p38, are involved in ER stress and oxidative stress (30), which indicates an association between the two. XBP-1 is a transcription factor in the UPR pathway, and previous studies have reported that XBP1 serves a protective role against oxidative stress $(21,31,32)$; therefore, XBP1 may be a potential link between the ER stress and oxidative stress pathways in cancer.

The imbalance between ROS and antioxidants is associated with a variety of pathogenic conditions, including cancer progression (33). A slight increase in ROS levels is beneficial to the development and progression of cancer, but excessive ROS production induces oxidative damage to proteins, lipids, RNA, DNA and other biological macromolecules in cancer cells, leading to cancer cell senescence and death (9). Therefore, tumor cells with increased endogenous ROS levels are likely to be more vulnerable to exogenous ROS-inducing agents. To the best of our knowledge, the present study was the first to reveal that XBP1 serves a role in controlling the intracellular ROS levels of SOC cells. XBP1 knockdown significantly enhanced endogenous ROS levels and increased sensitivity of SOC cells to oxidative stress, which indicated that knockdown of XBP1 combined with a ROS inducer may be applied in the treatment of ovarian cancer in the future. In the present study, the knockdown of XBP1 increased the protein expression levels of HMOX1. When cancer cells respond to oxidative stress, HMOX1 may reduce intracellular ROS by upregulating its expression. HMOX1 upregulation may occur due to the increased intracellular ROS level following XBP1 downregulation.

In the present study, the expression levels of the apoptosis-related proteins $\mathrm{p} 53$ and $\mathrm{Bcl} 2$ were not associated with XBP1 in SOC cells, but the phosphorylation level of p38 was associated with XBP1 expression. The p38 MAPK signaling pathway is a key signal transduction cascade, which controls the balance between cancer cell survival and death in response to microenvironmental stress $(34,35)$. The complexity of this regulation may determine the cell fate, i.e., survival or death, depending largely on the type and intensity of the stress, as well as the cell type (36). Accumulating evidence suggests that the p38 signaling pathway serves a dual role in various types of cancer. Wagner and Nebreda (37) suggested that p38 is associated with apoptosis in hepatoma cells and another study indicated that p38 is closely related to the chemoresistance of colorectal cancer (38). This dual role poses a challenge to the development of highly effective anticancer therapies targeting the p38 MAPK pathway. The present study demonstrated that the knockdown of XBP1 decreased the phosphorylation levels of the stress kinase p38. It is necessary to further explore the association between XBP1 and p38 and to elucidate the role of p38 in the development of SOC.

In conclusion, the results of the present study demonstrated that XBP1 is overexpressed in SOC and that knockdown of XBP1 significantly inhibited cell proliferation, which indicated that XBP1 may be a crucial transcription factor for the survival of ovarian cancer cells. In addition, XBP1 knockdown enhanced the sensitivity of SOC cells to oxidative stress through upregulation of endogenous ROS levels. In conclusion, XBP1 may be a candidate molecular target for inhibition of SOC cell growth, and may act synergistically with ROS inducers in anticancer treatment.

\section{Acknowledgements}

The authors would like to thank the Chinese Academy of Sciences Committee (Shanghai, China) for providing the SOC cell lines A2780 and HO8910.

\section{Funding}

This study was supported by the National Natural Science Foundation of China (grant nos. NSF-81572552, NSF-81772774 and NSF-81772808), the Cancer Research Program of National Cancer Center (grant no. NCC 2017A01), and the Science and Technology Commission of Shanghai Municipality (grant nos. 17411963500 and 17411951000).

\section{Availability of data and materials}

The datasets used and/or analyzed during the current study are available from the corresponding author on reasonable request.

\section{Authors' contributions}

The experiments were conceived and designed by LG and RQL. GHZ, JYK, MMC, QM and ALZ performed the experiments. SHX performed the statistical analysis. YCW, HZ, YiT and YuT analyzed the data. GHZ and JYK wrote the manuscript. LG, SHX and RQL revised the manuscript. All authors agreed to be accountable for all aspects of this work.

\section{Ethics approval and consent to participate}

Not applicable.

\section{Patient consent for publication}

Not applicable.

\section{Competing interests}

The authors declare that they have no competing interests.

\section{References}

1. Siegel RL, Miller KD and Jemal A: Cancer statistics, 2017. CA Cancer J Clin 67: 7-30, 2017.

2. Markman M: Current standards of care for chemotherapy of optimally cytoreduced advanced epithelial ovarian cancer. Gynecol Oncol 131: 241-245, 2013

3. Zhang H, Zhong A, Sun J, Chen M, Xie S, Zheng H, Wang Y, $\mathrm{Yu}$ Y, Guo L and Lu R: COPS5 inhibition arrests the proliferation and growth of serous ovarian cancer cells via the elevation of p27 level. Biochem Biophys Res Commun 493: 85-93, 2017.

4. Coscia F, Lengyel E, Duraiswamy J, Ashcroft B, Bassani-Sternberg M, Wierer M, Johnson A, Wroblewski K, Montag A, Yamada SD, et al: Multi-level proteomics identifies CT45 as a chemosensitivity mediator and immunotherapy target in ovarian cancer. Cell 175: 159-170.e16, 2018. 
5. Gentric G, Kieffer Y, Mieulet V, Goundiam O, Bonneau C, Nemati F, Hurbain I, Raposo G, Popova T, Stern MH, et al: PML-Regulated mitochondrial metabolism enhances chemosensitivity in human ovarian cancers. Cell Metab 29: 156-173.e10, 2019

6. Macintyre G, Goranova TE, De Silva D, Ennis D, Piskorz AM Eldridge M, Sie D, Lewsley LA, Hanif A, Wilson C, et al: Copy number signatures and mutational processes in ovarian carcinoma. Nat Genet 50: 1262-1270, 2018.

7. Schumacker PT: Reactive oxygen species in cancer cells: Live by the sword, die by the sword. Cancer Cell 10: 175-176, 2006.

8. Szatrowski TP and Nathan CF: Production of large amounts of hydrogen peroxide by human tumor cells. Cancer Res 51: 794-798, 1991 .

9. Zou Z, Chang H, Li H and Wang S: Induction of reactive oxygen species: An emerging approach for cancer therapy. Apoptosis 22: 1321-1335, 2017.

10. Pluchino LA, Choudhary S and Wang HC: Reactive oxygen species-mediated synergistic and preferential induction of cell death and reduction of clonogenic resistance in breast cancer cells by combined cisplatin and FK228. Cancer Lett 381: 124-132, 2016

11. Cao S, Xia M, Mao Y, Zhang Q, Donkor PO, Qiu F and Kang N: Combined oridonin with cetuximab treatment shows synergistic anticancer effects on laryngeal squamous cell carcinoma: Involvement of inhibition of EGFR and activation of reactive oxygen species-mediated JNK pathway. Int J Oncol 49: 2075-2087, 2016.

12. Yoshida H, Matsui T, Yamamoto A, Okada T and Mori K: XBP1 mRNA is induced by ATF6 and spliced by IRE1 in response to ER stress to produce a highly active transcription factor. Cell 107 881-891, 2001

13. Reimold AM, Iwakoshi NN, Manis J, Vallabhajosyula P, Szomolanyi-Tsuda E, Gravallese EM, Friend D, Grusby MJ, Alt $\mathrm{F}$ and Glimcher LH: Plasma cell differentiation requires the transcription factor XBP-1. Nature 412: 300-307, 2001.

14. Clauss IM, Gravallese EM, Darling JM, Shapiro F, Glimcher MJ and Glimcher LH: In situ hybridization studies suggest a role for the basic region-leucine zipper protein hXBP-1 in exocrine gland and skeletal development during mouse embryogenesis. Dev Dyn 197: 146-156, 1993.

15. Romero-Ramirez L, Cao H, Nelson D, Hammond E, Lee AH, Yoshida H, Mori K, Glimcher LH, Denko NC, Giaccia AJ, et al: XBP1 is essential for survival under hypoxic conditions and is required for tumor growth. Cancer Res 64: 5943-5947, 2004.

16. Chen X, Iliopoulos D, Zhang Q, Tang Q, Greenblatt MB, Hatziapostolou M, Lim E, Tam WL, Ni M, Chen Y, et al: XBP1 promotes triple-negative breast cancer by controlling the HIF1a pathway. Nature 508: 103-107, 2014.

17. Li H, Chen X, Gao Y, Wu J, Zeng F and Song F: XBP1 induces snail expression to promote epithelial-to-mesenchymal transition and invasion of breast cancer cells. Cell Signal 27: 82-89, 2015.

18. Gomez BP, Riggins RB, Shajahan AN, Klimach U, Wang A, Crawford AC, Zhu Y, Zwart A, Wang M and Clarke R: Human $\mathrm{X}$-box binding protein-1 confers both estrogen independence and antiestrogen resistance in breast cancer cell lines. FASEB J 21: 4013-4027, 2007.

19. Chen L, Li Q, She T, Li H, Yue Y, Gao S, Yan T, Liu S, Ma J and Wang Y: IRE1 $\alpha$-XBP1 signaling pathway, a potential therapeutic target in multiple myeloma. Leuk Res 49: 7-12, 2016.

20. Ojha $\mathrm{R}$ and Amaravadi RK: Targeting the unfolded protein response in cancer. Pharmacol Res 120: 258-266, 2017.

21. Liu Y, Zhang X, Liang Y, Yu H, Chen X, Zheng T, Zheng B, Wang L, Zhao L, Shi C and Zhao S: Targeting X box-binding protein-1 (XBP1) enhances sensitivity of glioma cells to oxidative stress. Neuropathol Appl Neurobiol 37: 395-405, 2011.

22. Liu Y, Adachi M, Zhao S, Hareyama M, Koong AC, Luo D, Rando TA, Imai K and Shinomura Y: Preventing oxidative stress: A new role for XBP1. Cell Death Differ 16: 847-857, 2009.
23. Cubillos-Ruiz JR, Silberman PC, Rutkowski MR, Chopra S, Perales-Puchalt A, Song M, Zhang S, Bettigole SE, Gupta D, Holcomb K, et al: ER stress sensor XBP1 controls anti-tumor immunity by disrupting dendritic cell homeostasis. Cell 161: $1527-1538,2015$

24. Livak KJ and Schmittgen TD: Analysis of relative gene expression data using real-time quantitative PCR and the 2(-Delta Delta C(T)) method. Methods 25: 402-408, 2001.

25. Mohamed E, Cao Y and Rodriguez PC: Endoplasmic reticulum stress regulates tumor growth and anti-tumor immunity: A promising opportunity for cancer immunotherapy. Cancer Immunol Immunother 66: 1069-1078, 2017.

26. Blais JD, Addison CL, Edge R, Falls T, Zhao H, Wary K, Koumenis C, Harding HP, Ron D, Holcik M and Bell JC: Perk-dependent translational regulation promotes tumor cell adaptation and angiogenesis in response to hypoxic stress. Mol Cell Biol 26: 9517-9532, 2006.

27. de la Cadena SG, Hernandez-Fonseca K, Camacho-Arroyo I and Massieu L: Glucose deprivation induces reticulum stress by the PERK pathway and caspase-7- and calpain-mediated caspase-12 activation. Apoptosis 19: 414-427, 2014.

28. Rouschop KM, Dubois LJ, Keulers TG, van den Beucken T, Lambin P, Bussink J, van der Kogel AJ, Koritzinsky $M$ and Wouters BG: PERK/eIF2 $\alpha$ signaling protects therapy resistant hypoxic cells through induction of glutathione synthesis and protection against ROS. Proc Natl Acad Sci USA 110: 4622-4627, 2013.

29. Schewe DM and Aguirre-Ghiso JA: ATF6alpha-Rheb-mTOR signaling promotes survival of dormant tumor cells in vivo. Proc Natl Acad Sci USA 105: 10519-10524, 2008.

30. Matsuzawa A, Nishitoh H, Tobiume K, Takeda K and Ichijo H: Physiological roles of ASK1-mediated signal transduction in oxidative stress- and endoplasmic reticulum stress-induced apoptosis: Advanced findings from ASK1 knockout mice. Antioxid Redox Signal 4: 415-425, 2002.

31. Chen TH, Chiang YH, Hou JN, Cheng CC, Sofiyatun E, Chiu CH and Chen WJ: XBP1-mediated BiP/GRP78 upregulation copes with oxidative stress in mosquito cells during dengue 2 virus infection. Biomed Res Int 2017: 3519158, 2017.

32. Martin D, Li Y, Yang J, Wang G, Margariti A, Jiang Z, Yu H, Zampetaki A, Hu Y, Xu Q and Zeng L: Unspliced X-box-binding protein 1 (XBP1) protects endothelial cells from oxidative stress through interaction with histone deacetylase 3. J Biol Chem 289: 30625-30634, 2014.

33. Matés JM, Pérez-Gómez C and Núñez de Castro I: Antioxidant enzymes and human diseases. Clin Biochem 32: 595-603, 1999.

34. Cuadrado A and Nebreda AR: Mechanisms and functions of $\mathrm{p} 38$ MAPK signalling. Biochem J 429: 403-417, 2010.

35. Cuenda A and Rousseau S: p38 MAP-kinases pathway regulation, function and role in human diseases. Biochim Biophys Acta 1773: 1358-1375, 2007.

36. Engelberg D: Stress-activated protein kinases-tumor suppressors or tumor initiators. Semin Cancer Biol 14: 271-282, 2004.

37. Wagner EF and Nebreda AR: Signal integration by JNK and p38 MAPK pathways in cancer development. Nat Rev Cancer 9 : 537-549, 2009.

38. Grossi V, Peserico A, Tezil T and Simone C: p38 $\alpha$ MAPK pathway: A key factor in colorectal cancer therapy and chemoresistance. World J Gastroenterol 20: 9744-9758, 2014.

This work is licensed under a Creative Common Attribution-NonCommercial-NoDerivatives 4.0 International (CC BY-NC-ND 4.0) License. 